\title{
Bounds for the Jensen Gap in terms of Power Means with Applications
}

\author{
Xuexiao You, ${ }^{1}$ Muhammad Adil Khan $\left(\mathbb{D},{ }^{2}\right.$ and Hamid Reza Moradi $(\mathbb{D})^{3}$ \\ ${ }^{1}$ School of Mathematics and Statistics, Hubei Normal University, Huangshi 435002, China \\ ${ }^{2}$ Department of Mathematics, University of Peshawar, Peshawar 25000, Pakistan \\ ${ }^{3}$ Department of Mathematics, Payame Noor University (PNU), P.O. Box 19395-4697, Tehran, Iran
}

Correspondence should be addressed to Hamid Reza Moradi; hrmoradi@mshdiau.ac.ir

Received 21 July 2021; Accepted 4 October 2021; Published 29 October 2021

Academic Editor: Muhammad Arif

Copyright (C) 2021 Xuexiao You et al. This is an open access article distributed under the Creative Commons Attribution License, which permits unrestricted use, distribution, and reproduction in any medium, provided the original work is properly cited.

Jensen's and its related inequalities have attracted the attention of several mathematicians due to the fact that Jensen's inequality has numerous applications in almost all disciplines of mathematics and in other fields of science. In this article, we propose new bounds for the difference of two sides of Jensen's inequality in terms of power means. An example has been presented for the importance and support of the main results. Related results have been given in quantum calculus. As consequences, improvements of quantum integral version of Hermite-Hadamard inequality have been derived. The obtained inequalities have been applied for some well-known inequalities such as Hermite-Hadamrd, Hölder, and power mean inequalities. Finally, some applications are given in information theory. The tools performed for obtaining the main results may be applied to obtain more results for other inequalities.

\section{Introduction}

There is no doubt that one of the most important classes of functions is the class of convex functions. The beauty of convex functions is due to its unique graphical representation, geometrical interpretation, and developments in the theory of inequalities. There are numerous applicable inequalities which have been established for this class of functions such as Jensen's, the Jensen-Steffensen, and majorization inequalities $[1,2]$. One of the most important and widely applicable inequalities which has attracted the attention of many mathematicians is the Jensen inequality [2, p. 43]. According to this inequality, if $\phi:\left[\alpha_{1}, \alpha_{2}\right] \longrightarrow \mathbb{R}$ is a convex function and $x_{i} \in\left[\alpha_{1}, \alpha_{2}\right], p_{i} \geq 0$ for each $i \in\{1,2, \cdots, n\}$ with $P_{n}:=$ $\sum_{i=1}^{n} p_{i}>0$, then

$$
\phi\left(\frac{\sum_{i=1}^{n} x_{i} p_{i}}{P_{n}}\right) \leq \frac{1}{P_{n}} \sum_{i=1}^{n} p_{i} \phi\left(x_{i}\right)
$$

The inequality in (1) flips when the function is concave.
The integral version of the Jensen inequality is presented in the following theorem [3].

Theorem 1. Suppose that $\left[\alpha_{1}, \alpha_{2}\right]$ is an interval and $g_{1}, g_{2}$ $:\left[a_{1}, a_{2}\right] \longrightarrow \mathbb{R}$ be functions such that $g_{1}(y) \in\left[\alpha_{1}, \alpha_{2}\right]$ for $y$ $\in\left[a_{1}, a_{2}\right]$. Let the function $\phi:\left[\alpha_{1}, \alpha_{2}\right] \longrightarrow \mathbb{R}$ be convex and the functions $g_{2}, g_{1} g_{2},\left(f \circ g_{1}\right) \cdot g_{2}$ be integrable. Also, assume that $g_{2}(y) \geq 0$ for all $y \in\left[a_{1}, a_{2}\right]$ and $\int_{a_{1}}^{a_{2}} g_{2}(y) d y=\Gamma>0$, then

$$
\phi\left(\frac{1}{\Gamma} \int_{a_{1}}^{a_{2}} g_{1}(y) g_{2}(y) d y\right) \leq \frac{1}{\Gamma} \int_{a_{1}}^{a_{2}}\left(\phi \circ g_{1}\right)(y) g_{2}(y) d y .
$$

This inequality has been utlized in Economics [4], Engineering [5], Optimization [6], Finance [7], Statistics [8], and Information theory $[3,9,10]$. For a particular convex function, this inequality provides many other inequalities such as AM-GM, Hölder and Ky Fan inequalities.

In the literature, discrepancy between the two sides of Jensen's inequality has been studied by several mathematicians in different directions which provides error bounds 
for certain approximations. In 2015, Costarelli and Spigler [11] studied the discrepancy between the right and left sides of Jensen's inequality by using convex functions from the class of $C^{2}$ functions as well as the class of merely Lipschitz continuous functions. Some illustrative examples are presented and compared the bounds with some existing bounds. In 2018, Pečarić et al. [12] focused to find the bounds for the difference of two sides of Jensen's inequality. They considered some Green convex functions and their related identities and derived bounds for the Jensen gap for the class of $C^{2}$ functions without using convexity condition. In particular, they have applied the Hölder inequality. Related discrete results have also been obtained, and several applications in information theory are presented. In 2020, Khan et al. [3] introduced a new method for the derivation of bounds using higher-order convex functions that is 4-convexity. First of all, they obtained an identity for the Jensen difference in terms of Green convex functions and double derivative of a function. Further, they obtained bounds by using 4-convexity and some properties of absolute function. Some examples are considered for their main results and compared with earlier bounds. Also, several applications for some well-known inequalities such as Hölder and Hermite-Hadmard inequalities have been given. At the end, several applications in information theory also presented. Related discrete results are given in [13]. In 2021, Khan et al. [14] further modified the method given in $[3,13]$ and derived several results for Jensen and related inequalities. In this method, they have used real weights and found the integrals of some functions which pertaining Green functions, in a very simple way with the help of the obtained identity for the Jensen gap. By virtue of this procedure, they were able to obtain bounds for the Jensen-Steffensen and converse of Jensen's inequalities. For more interesting results related to the celebrated Jensen's inequality, we recommend [15-17].

The main results of this manuscript utilize power means and its related inequalities; therefore, we want to mention them in the following part of this section. The following well-known power means and their monotonicity are given in $[18, \mathrm{p} .19]$ :

For two positive real $n$-tuples $\mathbf{x}=\left(x_{1}, \cdots, x_{n}\right)$ and $\mathbf{p}=$ $\left(p_{1}, \cdots, p_{n}\right)$, the power mean of order $\kappa \in \mathbb{R}$ is defined by

$$
M_{\kappa}(\mathbf{x} ; \mathbf{p})=\left\{\begin{array}{l}
\left(\frac{1}{P_{n}} \sum_{i=1}^{n} p_{i} x_{i}^{\kappa}\right)^{1 / \kappa}, \text { if } \kappa \neq 0, \\
\left(\prod_{i=1}^{n} x_{i}^{p_{i}}\right)^{1 / P_{n}}, \text { if } \kappa=0
\end{array}\right.
$$

where $P_{n}=\sum_{i=1}^{n} p_{i}$.

For the above $n$-tuples, the quasiarithmetic mean is defined by

$$
\tilde{M}_{h}(\mathbf{x} ; \mathbf{p})=h^{-1}\left(\frac{1}{P_{n}} \sum_{i=1}^{n} p_{i} h\left(x_{i}\right)\right)
$$

where $h$ is a strictly monotone and continuous function.
The integral power mean can be defined as follows: If $p$, $g:[a, b] \longrightarrow R^{+}$and $g$ are integrable functions, then the integral power mean of order $\kappa \in \mathbb{R}$ is defined by

$$
\bar{M}_{\kappa}(g ; p)=\left\{\begin{array}{l}
\left(\frac{1}{\int_{a}^{b} p(\omega) d \omega} \int_{a}^{b} p(\omega) g^{\kappa}(\omega) d \omega\right)^{1 / \kappa}, \text { if } \kappa \neq 0, \\
\exp \left(\frac{\int_{a}^{b} p(\omega) \log g(\omega) d \omega}{\int_{a}^{b} p(\omega) d \omega}\right), \text { if } \kappa=0 .
\end{array}\right.
$$

If $\kappa_{1} \leq \kappa_{2}$, then

$$
\begin{aligned}
& M_{\kappa_{1}}(\mathbf{x} ; \mathbf{p}) \leq M_{\kappa_{2}}(\mathbf{x} ; \mathbf{p}), \\
& \bar{M}_{\kappa_{1}}(g ; p) \leq \bar{M}_{\kappa_{2}}(g ; p) .
\end{aligned}
$$

The main aim of this paper is to obtain new interesting bounds for the discrepancy of the two sides of the Jensen inequality using new tools. The bounds pertain power means. We give an example, which shows that the bounds obtained in this paper are better than the earlier ones. We proved Jensen's inequality for quantum integrals and also derived its improvements. As applications, Hermite-Hadamard inequality and its improvements have been deduced for $q$-integrals. We also give applications for some well-known inequalities such as Hermite-Harmard, Hölder, power, and quasiarithmetic mean inequalities. At the end, we focused to give applications for Shannon-entropy, Csiszár, and Zipf-Mandelbrot entropy etc.

\section{Main Results}

We begin by presenting our first major finding.

Theorem 2. Let $\phi:[a, b] \longrightarrow \mathbb{R}$ be a convex function, $x_{i} \in$ $[a, b], p_{i}>0$ for $i=1,2, \cdots, n$ with $P_{n}=\sum_{i=1}^{n} p_{i}$. Then, for $s \geq$ 1 and $r \leq 1$, the following inequalities hold:

$$
M_{r}(y ; p) \leq \frac{1}{P_{n}} \sum_{i=1}^{n} p_{i} \phi\left(x_{i}\right)-\phi(\bar{x}) \leq M_{s}(y ; p),
$$

where $\bar{x}=\left(1 / P_{n}\right) \sum_{i=1}^{n} p_{i} x_{i}$ and $y=\left(y_{1}, y_{2}, \cdots, y_{n}\right)$ with $y_{i}=\phi($ $\left.x_{i}\right)-\phi(\bar{x})-\phi_{+}^{\prime}(\bar{x})\left(x_{i}-\bar{x}\right)$ for $i=1,2, \cdots, n$.

Proof. It is obvious that

$$
\begin{aligned}
\frac{1}{P_{n}} \sum_{i=1}^{n} p_{i} \phi\left(x_{i}\right)-\phi(\bar{x}) & =\frac{1}{P_{n}} \sum_{i=1}^{n} p_{i}\left(\phi\left(x_{i}\right)-\phi(\bar{x})-\phi_{+}^{\prime}(\bar{x})\left(x_{i}-\bar{x}\right)\right) \\
& =\frac{1}{P_{n}} \sum_{i=1}^{n} p_{i} y_{i} .
\end{aligned}
$$

Since the function $\phi$ is convex, therefore, we have

$$
y_{i}:=\phi\left(x_{i}\right)-\phi(\bar{x})-\phi_{+}^{\prime}(\bar{x})\left(x_{i}-\bar{x}\right) \geq 0
$$


Hence, by the power mean inequality (6), we have

$$
\frac{1}{P_{n}} \sum_{i=1}^{n} p_{i} y_{i} \leq M_{s}(y ; p), \text { for } s \geq 1,
$$

and similarly,

$$
M_{r}(y ; p) \leq \frac{1}{P_{n}} \sum_{i=1}^{n} p_{i} y_{i}, \text { for } r \leq 1
$$

Using (11) and (12) in (9), we obtain (8).

In the theorem below, we give an integral version of the above theorem.

Theorem 3. Consider the interval I and the convex function $\phi: I \longrightarrow \mathbb{R}$ and let $g, p:[a, b] \longrightarrow \mathbb{R}$ be integrable functions such that $g(\ell) \in I, p(\ell) \in \mathbb{R}^{+}$for all $\ell \in[a, b]$ and $P=\int_{a}^{b} p(\ell) d \ell$ . Then, for $s \geq 1$ and $r \leq 1$, we have

$$
\bar{M}_{r}(h ; p) \leq \frac{1}{P} \int_{a}^{b} p(\ell) \phi(g(\ell)) d \ell-\phi(\bar{g}) \leq \bar{M}_{s}(h ; p),
$$

where $h(\ell)=\phi(g(\ell))-\phi(\bar{g})-\phi^{\prime}{ }_{+}(\bar{g})(g(\ell)-\bar{g})$ with $\bar{g}=(1 /$ P) $\int_{a}^{b} p(\ell) g(\ell) d \ell, \ell \in[a, b]$.

The next main result is presented in the following theorem.

Theorem 4. Let $\phi:[a, b] \longrightarrow \mathbb{R}$ be a convex function, $x_{i} \in$ $[a, b]$ and $p_{i}>0$ for $i=1,2, \cdots, n$ with $P_{n}=\sum_{i=1}^{n} p_{i}$. Then, for $s \geq 1$ and $r \leq 1$, we have

$$
\frac{\bar{y}}{P_{n}} M_{r}(p ; y) \leq \frac{1}{P_{n}} \sum_{i=1}^{n} p_{i} \phi\left(x_{i}\right)-\phi(\bar{x}) \leq \frac{\bar{y}}{P_{n}} M_{s}(p ; y),
$$

where $y=\left(y_{1}, y_{2}, \cdots, y_{n}\right)$ with $y_{i}=\phi\left(x_{i}\right)-\phi(\bar{x})-\phi_{+}^{\prime}(\bar{x})\left(x_{i}\right.$ $-\bar{x})$ for $i=1,2, \cdots, n$ and $\bar{x}=\left(1 / P_{n}\right) \sum_{i=1}^{n} p_{i} x_{i}, \bar{y}=\sum_{i=1}^{n} y_{i}$.

Proof. By simple calculation, we can write

$$
\begin{aligned}
\frac{1}{P_{n}} \sum_{i=1}^{n} p_{i} \phi\left(x_{i}\right)-\phi(\bar{x}) & =\frac{1}{P_{n}} \sum_{i=1}^{n} p_{i}\left(\phi\left(x_{i}\right)-\phi(\bar{x})-\phi_{+}^{\prime}(\bar{x})\left(x_{i}-\bar{x}\right)\right) \\
& =\frac{1}{P_{n}} \sum_{i=1}^{n} p_{i} y_{i} .
\end{aligned}
$$

Since the function $\phi$ is convex, therefore, we have

$$
y_{i}:=\phi\left(x_{i}\right)-\phi(\bar{x})-\phi_{+}^{\prime}(\bar{x})\left(x_{i}-\bar{x}\right) \geq 0 .
$$

As $\bar{y}:=\sum_{i=1}^{n} y_{i}$, so from (15), we can write

$$
\frac{1}{P_{n}} \sum_{i=1}^{n} p_{i} \phi\left(x_{i}\right)-\phi(\bar{x})=\frac{\bar{y}}{P_{n}} \frac{\sum_{i=1}^{n} y_{i} p_{i}}{\bar{y}}
$$

Now, by considering the weights $y_{i}$ and applying power mean inequality (6), we have

$$
\frac{\sum_{i=1}^{n} y_{i} p_{i}}{\bar{y}} \leq M_{s}(p ; y), \text { for } s \geq 1,
$$

and similarly,

$$
\frac{\sum_{i=1}^{n} y_{i} p_{i}}{\bar{y}} \geq M_{r}(p ; y) \text {, for } r \leq 1
$$

Using (18) and (19) in (17), we obtain (14).

In the forthcoming theorem, we present the integral version of the above theorem.

Theorem 5. Consider $\phi: I \longrightarrow \mathbb{R}$ be a convex function and $p, g:[a, b] \longrightarrow \mathbb{R}$ be integrable functions such that $g(\omega) \in I$ ,$p(\omega) \in \mathbb{R}^{+}$for all $\omega \in[a, b]$ and $P=\int_{a}^{b} p(\omega) d \omega$. Then, for $s$ $\geq 1$ and $r \leq 1$, the following inequalities hold:

$$
\frac{\bar{h}}{P} \bar{M}_{r}(p ; h) \leq \frac{1}{P} \int_{a}^{b} p(\omega) \phi(g(\omega)) d \omega-\phi(\bar{g}) \leq \frac{\bar{h}}{P} \bar{M}_{s}(p ; h),
$$

where $\bar{g}=(1 / P) \int_{a}^{b} p(\omega) g(\omega) d \omega$ and $\bar{h}=\int_{a}^{b} h(\omega) d \omega$ with $h(\omega)=\phi(g(\omega))-\phi(\bar{g})-\phi_{+}^{\prime}(\bar{g})(g(\omega)-\bar{g}), \omega \in[a, b]$.

In the following example, we compare our new bound with earlier bounds of the Jensen gap.

Example 1. Let the functions $\phi, g:[0,1] \longrightarrow \mathbb{R}$ be defined by $\phi(x)=x^{4}, g(x)=x$ and $p(x)=1$ for all $x \in[0,1]$. Then,

$$
P=1, \bar{g}=\frac{1}{P} \int_{0}^{1} p(x) g(x) d x=\int_{0}^{1} x d x=\frac{1}{2}, \phi^{\prime}(\bar{g})=\frac{1}{2},
$$

and $h(x)=x^{4}-(x / 2)+(3 / 16), \bar{h}=\int_{0}^{1}\left(x^{4}-(x / 2)+(3 / 16)\right) d$ $x=0.1375$.

Now, we calculate the right hand side of (13) for $s=2$ :

$$
\bar{M}_{2}(h ; p)=\left(\int_{0}^{1}\left(x^{4}-\frac{x}{2}+\frac{3}{16}\right)^{2} d x\right)^{1 / 2}=0.2101 .
$$

For the same functions, the bound for the Jensen gap from inequality (5) in [3] is $a_{1}:=0.25$. Also, in bounds for the Jensen gap from the inequalities (6) and (11) in [11], we have $a_{2}=0.8748$ and $a_{3}=0.4998$, respectively. 
Hence, we concluded that $\bar{M}_{2}(h, p)=0.2101<a_{i}$ for $i$ $=1,2,3$. That is, for these functions, the bound obtained in $(13)$ is better than the earlier bound obtained in $[3,11]$.

As $\bar{M}_{s}(h ; p)$ is increasing with respect to $s$, therefore for more better estimate, we can find $\bar{M}_{s}(h, p)$ for $1<s<2$. For example, $\bar{M}_{1.5}(h ; p)$ is given by

$$
\begin{aligned}
\bar{M}_{1.5}(h ; p) & =\left(\int_{0}^{1}\left(x^{4}-\frac{x}{2}+\frac{3}{16}\right)^{1.5} d x\right)^{1 / 1.5} \\
& \approx 0.1753 \text {, that is } \bar{M}_{1.5}(h ; p)<\bar{M}_{2}(h ; p) .
\end{aligned}
$$

For inequality (20), we consider the above functions with $p(x)=x$. For these functions, the value of the Jensen difference is 0.1358 , and the value of the bound for the Jensen gap from the inequality (5) in [3] is: $b_{1}:=0.2074$. Now, we calculate the right hand side of (20).

$$
\begin{aligned}
P=0.2, \bar{h} & =\int_{0}^{1}\left(x^{4}-1.1854 x+0.5927\right) d x=0.2, \\
\frac{\bar{h}}{P} \bar{M}_{2}(p ; h) & =\frac{0.2}{0.5}\left(\frac{1}{0.2} \int_{0}^{1}\left(x^{4}-1.1854 x+0.5927\right) x^{2} d x\right)^{1 / 2} \\
& =0.1878 .
\end{aligned}
$$

So, $(\bar{h} / P) \bar{M}_{2}(p ; h)<b_{1}$. Similarly, we can take more better bound by considering $s=1 / 2$ that is

$$
\begin{aligned}
\frac{\bar{h}}{\bar{P}} \bar{M}_{1.5}(p ; h) & =\frac{0.2}{0.5}\left(\frac{1}{0.2} \int_{0}^{1}\left(x^{4}-1.1854 x+0.5927\right) x^{1.5} d x\right)^{1 / 1.5} \\
& =0.1634 .
\end{aligned}
$$

Hence, in this case, we concluded that the bound obtained in (20) is better than the earlier bound obtained in the inequality (5) in [3].

\section{Jensen's Type Inequalities in Quantum Calculus}

The $q$-calculus or quantum calculus deals with the study of calculus without utlizing the idea of limits. The popular mathematician Euler proposed the ponder $q$-calculus within the $18^{\text {th }}$ century, while he introduced the term $q$ in Newton's work of infinite series. Jackson has begun a symmetric study of $q$-calculus and presented $q$-definite integrals in twentieth century [19]. The field of $q$-calculus has various interesting applications in several branches of Physics, Mathematics, and in other areas $[20,21]$. This field has gotten extraordinary attention by numerous researchers, and a lot of research is devoted to this field. In [22], the authors defined $q$-analogue operator of Ruscheweyh type involving multivalent functions and derived several properties. By using the newly presented Harmonic $q$-Starlike class of functions, some important problems such as distortion limits, neces- sary and sufficient conditions, convolutions and convexity, and problems with partial sums have been studied in [23]. Srivastava et al. [24] applied the idea of a particular advanced convolution $q$-operator together with the concept of convolution and analyzed two new classes of meromorphically harmonic functions.

First, we give some preliminaries which are useful in our results. Throughout this section, $q$ belongs to $(0,1)$. Also, we assume that all the series which are used in this section are convergent.

Definition 6 (see [25]). Let $\psi:[a, b] \longrightarrow \mathbb{R}$ be a continuous function and $y \in[a, b]$. Then, the $q$-derivative of the function $\psi$ at $y$ is denoted by ${ }_{a} D_{q} \psi(y)$ and defined by

$$
\begin{aligned}
& { }_{a} D_{q} \psi(y)=\frac{\psi(y)-\psi(q y+(1-q) a)}{(1-q)(y-a)}, y \neq a, \\
& { }_{a} D_{q} \psi(a)=\lim _{y \longrightarrow a} D_{q} \psi(y) .
\end{aligned}
$$

We say that $\psi$ is $q$-differentiable on $[a, b]$ if ${ }_{a} D_{q} \psi(y)$ exists for all $y \in[a, b]$.

Definition 7 (see [25]). Let $\psi:[a, b] \longrightarrow \mathbb{R}$ be a continuous function. Then, the $q$-integral on $[a, b]$ is defined as

$$
\int_{a}^{y} \psi(x)_{a} d_{q} x=(1-q)(y-a) \sum_{k=0}^{\infty} q^{k} \psi\left(q^{k} y+\left(1-q^{k}\right) a\right),
$$

for $y \in[a, b]$. Moreover, if $c \in(a, y)$, then the $q$-integral on [ $a, b]$ is defined as

$$
\int_{c}^{y} \psi(x)_{a} d_{q} x=\int_{a}^{y} \psi(x)_{a} d_{q} x-\int_{a}^{c} \psi(x)_{a} d_{q} x .
$$

Remark 8. From Definitions 6 and 7, we make the following remarks:

(1) By taking $a=0$, the expression in (26) becomes the well-known $q$-derivative, $D_{q} \psi(w)$, of the function $\psi$ defined by

$$
D_{q} \psi(w)=\frac{\psi(y)-\psi(q y)}{(1-q) y}
$$

(2) Also, if $a=0$, then (27) reduces to the classical $q$ -integral of a function $\psi:[0, \infty) \longrightarrow \mathbb{R}$ defined by

$$
\int_{0}^{y} \psi(x)_{0} d_{q} x=(1-q) y \sum_{k=0}^{\infty} q^{k} \psi\left(q^{k} y\right)
$$

Now, we present Jensen's inequality for $q$-integrals. 
Theorem 9. Let $\phi: I \longrightarrow \mathbb{R}$ be a continuous convex function defined on the interval $I$ and $\psi:[a, b] \longrightarrow I$ be a continuous function. Then,

$$
\phi\left(\frac{1}{b-a} \int_{a}^{b} \psi(x)_{a} d_{q} x\right) \leq \frac{1}{b-a} \int_{a}^{b} \phi(\psi(x))_{a} d_{q} x .
$$

Proof. From the convexity of $\phi$, we have

$$
\phi(x)-\phi(y) \geq \phi_{+}^{\prime}(y)(x-y), \text { for all } x, y \in I
$$

Taking $x=\psi\left(q^{k} b+\left(1-q^{k}\right) a\right)$ for $k=0,1,2, \cdots$ and $y=$ $\bar{y}=(1-q) \sum_{k=0}^{\infty} q^{k} \psi\left(q^{k} b+\left(1-q^{k}\right) a\right)=(1 /(b-a) b-a) \int_{a}^{b} \psi$ $(x)_{a} d_{q} x$, in (32), we obtain

$$
\begin{aligned}
& \phi\left(\psi\left(q^{k} b+\left(1-q^{k}\right) a\right)\right)-\phi(\bar{y}) \\
& \quad \geq \phi_{+}^{\prime}(\bar{y})\left(\psi\left(q^{k} b+\left(1-q^{k}\right) a\right)-\bar{y}\right) .
\end{aligned}
$$

Multiplying both sides of (33) by $(1-q) q^{k}$ and then taking summation over $k$, we get

$$
\begin{array}{r}
(1-q) \sum_{k=0}^{\infty} q^{k} \phi\left(\psi\left(q^{k} b+\left(1-q^{k}\right) a\right)\right)-\phi(\bar{y}) \geq \phi_{+}^{\prime}(\bar{y}) \\
\cdot\left((1-q) \sum_{k=0}^{\infty} q^{k} \psi\left(q^{k} b+\left(1-q^{k}\right) a\right)-\bar{y}\right) .
\end{array}
$$

Since $\quad \bar{y}=(1-q) \sum_{k=0}^{\infty} q^{k} \psi\left(q^{k} b+\left(1-q^{k}\right) a\right), \quad$ therefore, from (34), we have

$$
(1-q) \sum_{k=0}^{\infty} q^{k} \phi\left(\psi\left(q^{k} b+\left(1-q^{k}\right) a\right)\right)-\phi(\bar{y}) \geq 0
$$

which is equivalent to (31).

As a consequence of the above theorem, we deduce Hermite-Hadamard inequality for quantum integral. This inequality has been proved in $[26,27]$.

Corollary 10. Let $\phi:[a, b] \longrightarrow \mathbb{R}$ be a continuous convex function. Then,

$$
\phi\left(\frac{b+a q}{1+q}\right) \leq \frac{1}{b-a} \int_{a}^{b} \phi(x)_{a} d_{q} x .
$$

Proof. If $\psi(x)=x$, then

$$
\begin{aligned}
\int_{a}^{b} \psi(x)_{a} d_{q} x & =\int_{a}^{b} x_{a} d_{q} x=(1-q)(b-a) \sum_{k=0}^{\infty} q^{k}\left(q^{k} b+\left(1-q^{k}\right) a\right) \\
& =(1-q)(b-a)\left(b \sum_{k=0}^{\infty} q^{2 k}+a \sum_{k=0}^{\infty} q^{k}-a \sum_{k=0}^{\infty} q^{2 k}\right) \\
& =(1-q)(b-a)\left(\frac{b}{1-q^{2}}+\frac{a}{1-q}-\frac{a}{1-q^{2}}\right) \\
& =\frac{(b+a q)(b-a)}{1+q} .
\end{aligned}
$$

Hence, using $\psi(x)=x$ in (31), we obtain (36).

Now, we give improvement of quantum integral version of Jensen's inequality in terms of means.

Theorem 11. Let $\phi: I \longrightarrow \mathbb{R}$ be a continuous convex function defined on the interval I and $\psi:[a, b] \longrightarrow I$ be a continuous function. Then, for $s \geq 1$ and $r \leq 1$, the following inequalities hold:

$$
\begin{aligned}
& \left((1-q) \sum_{k=0}^{\infty} q^{k} z_{k}^{s}\right)^{1 / s} \\
& \leq \frac{1}{b-a} \int_{a}^{b} \phi(\psi(x))_{a} d_{q} x-\phi\left(\frac{1}{b-a} \int_{a}^{b} \psi(x)_{a} d_{q} x\right) \\
& \leq\left((1-q) \sum_{k=0}^{\infty} q^{k} z_{k}^{r}\right)^{1 / r},
\end{aligned}
$$

where $z_{k}:=\phi\left(\psi\left(q^{k} b+\left(1-q^{k}\right) a\right)\right)-\phi(\bar{y})-\phi^{\prime}{ }_{+}(\bar{y})\left(\psi\left(q^{k} b+\right.\right.$ $\left.\left.\left(1-q^{k}\right) a\right)-\bar{y}\right)$ with $\bar{y}=(1 /(b-a) b-a) \int_{a}^{b} \psi(x)_{a} d_{q} x$.

Proof. From the proof of Theorem 9, we see that

$$
\begin{aligned}
& \frac{1}{b-a} \int_{a}^{b} \phi(\psi(x))_{a} d_{q} x-\phi\left(\frac{1}{b-a} \int_{a}^{b} \psi(x)_{a} d_{q} x\right) \\
& =(1-q) \sum_{k=0}^{\infty} q^{k}\left(\phi\left(\psi\left(q^{k} b+\left(1-q^{k}\right) a\right)\right)\right. \\
& \left.\quad-\phi(\bar{y})-\phi_{+}^{\prime}(\bar{y})\left(\psi\left(q^{k} b+\left(1-q^{k}\right) a\right)-\bar{y}\right)\right) .
\end{aligned}
$$

By convexity of $\phi$, we have $z_{k}:=\phi\left(\psi\left(q^{k} b+\left(1-q^{k}\right) a\right)\right)$ $-\phi(\bar{y})-\phi_{+}^{\prime}(\bar{y})\left(\psi\left(q^{k} b+\left(1-q^{k}\right) a\right)-\bar{y}\right) \geq 0$. Also, as $q \in(0$, 1) so by geometric series we have $\sum_{k=0}^{\infty} q^{k}=1 /(1-q)$ that is $(1-q) \sum_{k=0}^{\infty} q^{k}=1 /(1-q)=1$ and hence $(1-q) \sum_{k=0}^{\infty} q^{k} z_{k}$ is the arithmetic mean. Now, by using power mean inequality in (39), we obtain 


$$
\begin{gathered}
\frac{1}{b-a} \int_{a}^{b} \phi(\psi(x))_{a} d_{q} x-\phi\left(\frac{1}{b-a} \int_{a}^{b} \psi(x)_{a} d_{q} x\right) \\
=(1-q) \sum_{k=0}^{\infty} q^{k} z_{k} \leq\left((1-q) \sum_{k=0}^{\infty} q^{k} z_{k}^{r}\right)^{1 / r} .
\end{gathered}
$$

Similarly, we can prove the left inequality in (42).

As an application of the above theorem, we deduce improvement of quantum integral version of HermiteHadamard inequality.

Corollary 12. Let $\phi:[a, b] \longrightarrow \mathbb{R}$ be a continuous convex function. Then, for $s \geq 1$ and $r \leq 1$, the following inequalities hold:

$$
\begin{aligned}
\left((1-q) \sum_{k=0}^{\infty} q^{k} t_{k}^{s}\right)^{1 / s} & \leq \frac{1}{b-a} \int_{a}^{b} \phi(x)_{a} d_{q} x-\phi\left(\frac{b+a q}{1+q}\right) \\
& \leq\left((1-q) \sum_{k=0}^{\infty} q^{k} t_{k}^{r}\right)^{1 / r} .
\end{aligned}
$$

where $t_{k}:=\phi\left(q^{k} b+\left(1-q^{k}\right) a\right)-\phi(b+a q / 1+q)-\phi_{+}^{\prime}(b+a q$ $/ 1+q)\left(q^{k} b+\left(1-q^{k}\right) a-b+a q / 1+q\right)$.

Proof. Using $\psi(x)=x$ in (42), we obtain (41).

In the following theorem, we present another improvement of quantum integral version of Jensen's inequality in terms of means.

Theorem 13. Let $\phi: I \longrightarrow \mathbb{R}$ be a continuous convex function defined on the interval I and $\psi:[a, b] \longrightarrow I$ be a continuous function. Then, for $s \geq 1$ and $r \leq 1$, the following inequalities hold:

$$
\begin{aligned}
(1 & -q) \sum_{k=0}^{\infty} z_{k}\left(\frac{\sum_{k=0}^{\infty} z_{k} q^{k s}}{\sum_{k=0}^{\infty} z_{k}}\right)^{1 / s} \\
& \leq \frac{1}{b-a} \int_{a}^{b} \phi(\psi(x))_{a} d_{q} x-\phi\left(\frac{1}{b-a} \int_{a}^{b} \psi(x)_{a} d_{q} x\right) \\
& \leq(1-q) \sum_{k=0}^{\infty} z_{k}\left(\frac{\sum_{k=0}^{\infty} z_{k} q^{k r}}{\sum_{k=0}^{\infty} z_{k}}\right)^{1 / r},
\end{aligned}
$$

where $z_{k}:=\phi\left(\psi\left(q^{k} b+\left(1-q^{k}\right) a\right)\right)-\phi(\bar{y})-\phi^{\prime}{ }_{+}(\bar{y})\left(\psi\left(q^{k} b+\right.\right.$ $\left.\left.\left(1-q^{k}\right) a\right)-\bar{y}\right)$ with $\bar{y}=(1 /(b-a) b-a) \int_{a}^{b} \psi(x)_{a} d_{q} x$.

Proof. The proof can be given by a similar way as the proof of Theorem 11.
As an application of Theorem 13, we deduce improvement of quantum integral version of Hermite-Hadamard inequality.

Corollary 14. Let $\phi:[a, b] \longrightarrow \mathbb{R}$ be a continuous convex function. Then, for $s \geq 1$ and $r \leq 1$, the following inequalities hold:

$$
\begin{aligned}
\left((1-q) \sum_{k=0}^{\infty} q^{k} t_{k}^{s}\right)^{1 / s} & \leq \frac{1}{b-a} \int_{a}^{b} \phi(x)_{a} d_{q} x-\phi\left(\frac{b+a q}{1+q}\right) \\
& \leq(1-q) \sum_{k=0}^{\infty} q^{k}\left(\frac{\sum_{k=0}^{\infty} q^{k r} t_{k}^{r}}{\sum_{k=0}^{\infty} q^{k}}\right)^{1 / r}
\end{aligned}
$$

where $t_{k}:=\phi\left(q^{k} b+\left(1-q^{k}\right) a\right)-\phi((b+a q) /(1+q))-\phi_{+}^{\prime}((b$ $+a q) /(1+q))\left(q^{k} b+\left(1-q^{k}\right) a-((b+a q) /(1+q))\right)$.

Proof. Using $\psi(x)=x$ in (42), we obtain (43).

\section{Applications for Some Well- Known Inequalities}

In this section, we demonstrate improvements of some wellknown inequalities. We start with the Hermite-Hadamard inequality.

Corollary 15. Let $\phi:[a, b] \longrightarrow \mathbb{R}$ be a convex function and $s \geq 1, r \leq 1$, then

$$
\bar{M}_{r}(\psi, I) \leq \frac{1}{b-a} \int_{a}^{b} \phi(x) d x-\phi\left(\frac{a+b}{2}\right) \leq \bar{M}_{s}(\psi, I)
$$

where $\psi(x)=\phi(x)-\phi((a+b) / 2)-\phi_{+}^{\prime}((a+b) / 2)(x-((a+$ $b) / 2)$ ) and $I(x)=1$ is the constant function.

Proof. Applying Theorem 5 for $p(\omega)=1, g(\omega)=\omega$, we obtain (44).

In the corollary below, we give improvements of power means inequality.

Corollary 16. Let $\mathbf{x}=\left(x_{1}, \cdots, x_{n}\right)$ and $\mathbf{p}=\left(p_{1}, \cdots, p_{n}\right)$ be positive $n$-tuples with $P_{n}=\sum_{i=1}^{n} p_{i}$ and $s, r, k_{1}, k_{2} \in \mathbb{R}$ such that $s$ $\geq 1, r \leq 1$ and $k_{1} \leq k_{2}$.

(i) If $k_{2} \neq 0$ and $k_{1}<0$, then

$$
M_{r}(w, p) \leq M_{k_{1}}^{k_{1}}(x, p)-M_{k_{2}}^{k_{1}}(x, p) \leq M_{s}(w, p)
$$

(ii) If $k_{1}>0$, then the reverse inequalities hold in (45) 
(iii) If $k_{1} \neq 0$ and $k_{2} \geq 0$, then

$$
M_{r}(z, p) \leq M_{k_{2}}^{k_{2}}(x, p)-M_{k_{1}}^{k_{2}}(x, p) \leq M_{s}(z, p)
$$

(iv) If $k_{2}<0$, then the reverse inequalities hold in (46)

where $w=\left(w_{1}, w_{2}, \cdots, w_{n}\right)$ with $w_{i}=x_{i}^{k_{1}}+\left(\left(k_{1}-k_{2}\right) / k_{2}\right)$ $M_{k_{2}}^{k_{1}}(x, p)-\left(k_{1} / k_{2}\right) x_{i}^{k_{2}} M_{k_{2}}^{k_{1}-k_{2}}(x, p)$ and $z=\left(z_{1}, z_{2}, \cdots, z_{n}\right)$ with $z_{i}=x_{i}^{k_{2}}+\left(\left(k_{2}-k_{1}\right) / k_{1}\right) M_{k_{2}}^{k_{1}}(x, p)-\left(k_{2} / k_{1}\right) x_{i}^{k_{1}} M_{k_{1}}^{k_{2}-k_{1}}(x$, p) for $i=1,2, \cdots, n$.

Proof.

(i) If $k_{1}<0, k_{1} \leq k_{2}$ and $\phi(x)=x^{k_{1} / k_{2}}$, then $\phi$ is convex. Therefore, applying Theorem 4 for this function and $x_{i} \longrightarrow x_{i}^{k_{2}}$, we obtain (45)

(ii) If $k_{1}>0$, then $\phi(x)=x^{k_{1} / k_{2}}$ is a concave function, so applying Theorem 4 for concave function, we deduce the reverse inequality in (45)

(iii) If $k_{1} \neq 0, k_{2} \geq 0$ and $\phi(x)=x^{k_{2} / k_{1}}$, then $\phi$ is convex. Therefore, applying Theorem 4 for this function and $x_{i} \longrightarrow x_{i}^{k_{1}}$, we obtain (46)

Similarly, we can prove the reverse inequality in (46).

Corollary 17. Let $\mathbf{p}=\left(p_{1}, \cdots, p_{n}\right)$ and $\mathbf{x}=\left(x_{1}, \cdots, x_{n}\right)$ be positive $n$-tuples with $P_{n}=\sum_{i=1}^{n} p_{i}$ and $s, r, \in \mathbb{R}$ such that $s \geq 1, r$ $\leq 1$. Let the function $h$ be monotone continuous function and $f \circ h^{-1}$ be convex function, then

$$
M_{r}(\bar{z}, p) \leq \frac{1}{P_{n}} \sum_{i=1}^{n} p_{i} f\left(x_{i}\right)-f\left(\tilde{M}_{h}(x, p)\right) \leq M_{s}(\bar{z}, p),
$$

where $\bar{z}=\left(\bar{z}_{1}, \bar{z}_{2}, \cdots, \bar{z}_{n}\right)$ with $\bar{z}_{i}=f\left(x_{i}\right)-f\left(\tilde{M}_{h}(x, p)\right)-(f \circ$ $\left.h^{-1}\right)^{\prime}{ }_{+}\left(\left(1 / P_{n}\right) \sum_{i=1}^{n} p_{i} h\left(x_{i}\right)\right)\left(h\left(x_{i}\right)-\left(1 / P_{n}\right) \sum_{i=1}^{n} p_{i} h\left(x_{i}\right)\right)$ for $i=$ $1,2, \cdots, n$.

Proof. The inequality (47) can be obtained by using Theorem 2 for $\phi \longrightarrow f \circ h^{-1}$ and $x_{i} \longrightarrow h\left(x_{i}\right)$ for $i=1,2, \cdots, n$.

The following two results are devoted to improvements of Hölder inequality.

Proposition 18. Let $\left(\gamma_{1}, \gamma_{2}, \cdots, \gamma_{n}\right),\left(\eta_{1}, \eta_{2}, \cdots, \eta_{n}\right)$ be positive $n$-tuples and $s, l, m \in \mathbb{R}$ be such that $s \geq 1$ and $l, m>1$ with $(1 / l)+(1 / m)=1$, then

$$
\begin{aligned}
& \left(\sum_{i=1}^{n} \eta_{i}^{m}\right)^{1 / m}\left(\sum_{i=1}^{n} \gamma_{i}^{l}\right)^{1 / l}-\sum_{i=1}^{n} \eta_{i} \gamma_{i} \\
& \leq\left(\sum_{i=1}^{n} \gamma_{i}^{l}\right)^{1-1 / m s}\left(\sum _ { i = 1 } ^ { n } \gamma _ { i } ^ { l } \left[\eta_{i}^{m} \zeta_{i}^{-l}-\left(\frac{\sum_{i=1}^{n} \gamma_{i} \eta_{i}}{\sum_{i=1}^{n} \gamma_{i}^{l}}\right)^{m}\right.\right. \\
& \left.\left.\quad-m\left(\frac{\sum_{i=1}^{n} \gamma_{i} \eta_{i}}{\sum_{i=1}^{n} \gamma_{i}^{l}}\right)^{m-1}\left(\eta_{i} \gamma_{i}^{-l / m}-\frac{\sum_{i=1}^{n} \eta_{i} \gamma_{i}}{\sum_{i=1}^{n} \gamma_{i}^{l}}\right)\right]^{s}\right)^{1 / m s} .
\end{aligned}
$$

Proof. Let $\phi(z)=z^{m}$, then clearly $\phi$ is convex for $z>0$ and $m>1$, therefore, using the right inequality in (8) for $\phi(z)$ $=z^{m}, p_{i}=\gamma_{i}^{l}$, and $x_{i}=\eta_{i} \gamma_{i}^{-l / m}$, and then simplifying, we derive that

$$
\begin{gathered}
\left(\left(\sum_{i=1}^{n} \eta_{i}^{m}\right)\left(\sum_{i=1}^{n} \gamma_{i}^{l}\right)^{m-1}-\left(\sum_{i=1}^{n} \eta_{i} \gamma_{i}\right)^{m}\right)^{1 / m} \\
\leq\left(\sum_{i=1}^{n} \gamma_{i}^{l}\right)^{1-1 / m s}\left(\sum _ { i = 1 } ^ { n } \gamma _ { i } ^ { l } \left[\eta_{i}^{m} \zeta_{i}^{-l}-\left(\frac{\sum_{i=1}^{n} \gamma_{i} \eta_{i}}{\sum_{i=1}^{n} \gamma_{i}^{l}}\right)^{m}\right.\right. \\
\left.\left.\quad-m\left(\frac{\sum_{i=1}^{n} \gamma_{i} \eta_{i}}{\sum_{i=1}^{n} \gamma_{i}^{l}}\right)^{m-1}\left(\eta_{i} \gamma_{i}^{-l / m}-\frac{\sum_{i=1}^{n} \eta_{i} \gamma_{i}}{\sum_{i=1}^{n} \gamma_{i}^{l}}\right)\right]^{s}\right)^{1 / m s} .
\end{gathered}
$$

Since the inequality $k_{1}^{\eta}-k_{2}^{\eta} \leq\left(k_{1}-k_{2}\right)^{\eta}$ holds for $\eta \in[0$, 1] and $0 \leq k_{2} \leq k_{1}$, therefore by putting $k_{1}=\left(\sum_{i=1}^{n} \eta_{i}^{m}\right)$ $\left(\sum_{i=1}^{n} \gamma_{i}^{l}\right)^{m-1}, k_{2}=\left(\sum_{i=1}^{n} \eta_{i} \gamma_{i}\right)^{m}$, and $\eta=1 / m$, we obtain

$$
\begin{aligned}
& \left(\sum_{i=1}^{n} \eta_{i}^{m}\right)^{1 / m}\left(\sum_{i=1}^{n} \gamma_{i}^{l}\right)^{1 / l}-\sum_{i=1}^{n} \eta_{i} \gamma_{i} \\
& \leq\left(\left(\sum_{i=1}^{n} \eta_{i}^{m}\right)\left(\sum_{i=1}^{n} \gamma_{i}^{l}\right)^{m-1}-\left(\sum_{i=1}^{n} \eta_{i} \gamma_{i}\right)^{m}\right)^{1 / m} .
\end{aligned}
$$

Now, (49) and (50) give (48).

Corollary 19. Let $\left(\gamma_{1}, \gamma_{2}, \cdots, \gamma_{n}\right),\left(\eta_{1}, \eta_{2}, \cdots, \eta_{n}\right)$ be positive $n$ -tuples and let $s, r, l, m \in \mathbb{R}$ be such that $0<l<1, m=l /(l-1)$ and $s \geq 1, r \leq 1$, then

$$
\begin{aligned}
M_{r}\left(j, \boldsymbol{\eta}^{m}\right) \sum_{i=1}^{n} \eta_{i}^{m} & \leq \sum_{i=1}^{n} \eta_{i} \gamma_{i}-\left(\sum_{i=1}^{n} \eta_{i}^{m}\right)^{1 / m}\left(\sum_{i=1}^{n} \gamma_{i}^{l}\right)^{1 / l} \\
& \leq M_{s}\left(j, \boldsymbol{\eta}^{m}\right) \sum_{i=1}^{n} \eta_{i}^{m}
\end{aligned}
$$

where $\boldsymbol{\eta}^{m}=\left(\eta_{1}^{m}, \eta_{2}^{m}, \cdots, \eta_{n}^{m}\right)$ and $j=\left(j_{1}, j_{2}, \cdots, j_{n}\right)$ with $j_{i}=$ $\gamma_{i} \eta_{i}^{-m / l}-\left(\sum_{i=1}^{n} \gamma_{i}^{l} / \sum_{i=1}^{n} \eta_{i}^{m}\right)^{1 / l}-(1 / l)\left(\sum_{i=1}^{n} \gamma_{i}^{l} / \sum_{i=1}^{n} \eta_{i}^{m}\right)^{(1 / l)-1}\left(\gamma_{i}\right.$ $\left.\eta_{i}^{l-m}-\left(\sum_{i=1}^{n} \gamma_{i}^{l} / \sum_{i=1}^{n} \eta_{i}^{m}\right)\right)$.

Proof. As the function $\phi(x)=x^{1 / l}$ is convex for $x>0, l \in(0$, $1)$. So by using (8) for $\phi(x)=x^{1 / l}, p_{i}=\eta_{i}^{m}$, and $x_{i}=\gamma_{i}^{l} \eta_{i}^{-m}$, we get (51). 
Remark 20. Similarly, we can present applications of the second main results. Also, we can give integral version of the above results.

\section{Applications in Information Theory}

Information theory studies how to measure, store, and transmit digital information. The field of information theory was initially entrenched by Hartley's work in 1920 and got worldwide attention by the work of Shannon in 1940s. The field of information theory is in close collaboration with probability theory, statistical mechanics, information, and electrical engineering. A fundamental measure in information theory is entropy that measures the uncertainty involved in the occurrence of a random process. After Shannon's work, the field attracted the concentration of various scientists and got inflated. Different entropy functional have been introduced that can be elaborated as generalized entropies.

Definition 21. The Csiszár $f$-divergence for two positive $n$ -tuples $\mathbf{l}=\left(l_{1}, l_{2}, \cdots, l_{n}\right), \mathbf{m}=\left(m_{1}, m_{2}, \cdots, m_{n}\right)$ is defined by

$$
C_{f}(\mathbf{l}, \mathbf{m})=\sum_{i=1}^{n} m_{i} f\left(\frac{l_{i}}{m_{i}}\right),
$$

where $f:(0, \infty) \longrightarrow \mathbb{R}$ is a convex function.

Theorem 22. Let $\mathbf{l}=\left(l_{1}, l_{2}, \cdots, l_{n}\right), \mathbf{m}=\left(m_{1}, m_{2}, \cdots, m_{n}\right)$ be positive $n$-tuples and $f:(0, \infty) \longrightarrow \mathbb{R}$ be a convex function. If $s \geq 1$ and $r \leq 1$, then

$$
\begin{aligned}
M_{r}(z, m) \sum_{i=1}^{n} m_{i} & \leq C_{f}(\mathbf{l}, \mathbf{m})-f\left(\frac{\sum_{i=1}^{n} l_{i}}{\sum_{i=1}^{n} m_{i}}\right) \sum_{i=1}^{n} m_{i} \\
& \leq M_{s}(z, m) \sum_{i=1}^{n} m_{i},
\end{aligned}
$$

where $z=\left(z_{1}, z_{2}, \cdots, z_{n}\right)$ with $z_{i}=f\left(l_{i} / m_{i}\right)-f\left(\sum_{i=1}^{n} l_{i} / \sum_{i=1}^{n} m_{i}\right)$ $-f^{\prime}{ }_{+}\left(\sum_{i=1}^{n} l_{i} / \sum_{i=1}^{n} m_{i}\right)\left(\left(l_{i} / m_{i}\right)-\left(\sum_{i=1}^{n} l_{i} / \sum_{i=1}^{n} m_{i}\right)\right)$ for $i=1,2$, $\cdots, n$.

Proof. Using (8) for $\phi=f, x_{i}=l_{i} / m_{i}$, and $p_{i}=m_{i}$ for $i \in\{1$, $2, \cdots, n\}$, we obtain (53).

Definition 23 (Shannon entropy). Let $\mathbf{q}=\left(q_{1}, q_{2}, \cdots, q_{n}\right)$ be positive probability distribution, then the Shannon entropy is defined by

$$
S(\mathbf{q})=-\sum_{i=1}^{n} q_{i} \log q_{i}
$$

Corollary 24. Let $\mathbf{m}=\left(m_{1}, m_{2}, \cdots, m_{n}\right)$ be a positive probability distribution. If $s \geq 1$ and $r \leq 1$, then

$$
M_{r}(z, m) \leq \log n-S(\mathbf{m}) \leq M_{s}(z, m)
$$

where $z=\left(z_{1}, z_{2}, \cdots, z_{n}\right)$ with $z_{i}=\log m_{i}+\log n+\left(1 / n m_{i}\right)$ -1 for $i=1,2, \cdots, n$.
Proof. Taking $f(\xi)=-\log \xi, \xi \in(0, \infty), l_{i}=1$, for each $i \in\{$ $1,2, \cdots, n\}$, in (53), we obtain (55).

Corollary 25. Let $\mathbf{m}=\left(m_{1}, m_{2}, \cdots, m_{n}\right), \mathbf{l}=\left(l_{1}, l_{2}, \cdots, l_{n}\right)$ be positive $n$-tuples with $\sum_{i=1}^{n} l_{i}=1$. If $s \geq 1$ and $r \leq 1$, then

$$
\begin{aligned}
M_{r}(z, m) \sum_{i=1}^{n} m_{i} & \leq-S(\mathbf{l})-\sum_{i=1}^{n} l_{i} \log m_{i}+\log \left(\sum_{i=1}^{n} m_{i}\right) \\
& \leq M_{s}(z, m) \sum_{i=1}^{n} m_{i}
\end{aligned}
$$

where $z=\left(z_{1}, z_{2}, \cdots, z_{n}\right)$ with $z_{i}=\left(l_{i} / m_{i}\right) \log \left(l_{i} / m_{i}\right)-(1 /$ $\left.\sum_{i=1}^{n} m_{i}\right) \log \left(1 / \sum_{i=1}^{n} m_{i}\right)-\left(1-\log \left(\sum_{i=1}^{n} m_{i}\right)\right)\left(\left(l_{i} / m_{i}\right)-(1 /\right.$ $\left.\left.\sum_{i=1}^{n} m_{i}\right)\right)$ for $i=1,2, \cdots, n$.

In particular, if $m_{i}=1$ for each $i=1,2, \cdots, n$, then

$$
n M_{r}(z, i) \leq-S(\mathbf{l})-\frac{\log n}{n} \leq n M_{s}(z, i)
$$

where $i=(1,1, \cdots, 1)$ and $z=\left(z_{1}, z_{2}, \cdots, z_{n}\right)$ with $z_{i}=l_{i} \log l_{i}$ $+(1 / n) \log n-(1-\log n)\left(l_{i}-(1 / n)\right)$ for $i=1,2, \cdots, n$.

Proof. Taking $f(x)=x, x \in(0, \infty)$, for each $i \in\{1,2, \cdots, n\}$, in (53), we obtain (56).

Definition 26. The Kullback-Leibler divergence for two positive probability distributions $\mathbf{l}=\left(l_{1}, l_{2}, \cdots, l_{n}\right)$ and $\mathbf{m}=$ $\left(m_{1}, m_{2}, \cdots, m_{n}\right)$ is defined by

$$
K_{d}(\mathbf{l}, \mathbf{m})=\sum_{i=1}^{n} l_{i} \log \left(\frac{l_{i}}{m_{i}}\right) .
$$

Corollary 27. Let $\mathbf{l}=\left(l_{1}, l_{2}, \cdots, l_{n}\right), \mathbf{m}=\left(m_{1}, m_{2}, \cdots, m_{n}\right)$ be positive probability distributions, then for $s \geq 1$ and $r \leq 1$, we have

$$
M_{r}(z, m) \leq K_{d}(\mathbf{l}, \mathbf{m}) \leq M_{s}(z, m)
$$

where $z=\left(z_{1}, z_{2}, \cdots, z_{n}\right)$ with $z_{i}=\left(l_{i} / m_{i}\right) \log \left(l_{i} / m_{i}\right)-2\left(\left(l_{i} /\right.\right.$ $\left.m_{i}\right)-1$ ) for $i=1,2, \cdots, n$.

Proof. Taking $f(x)=x \log x, x \in(0, \infty)$, in (53), we obtain (59).

Corollary 28. Assume that all the assumptions of Corollary 27 hold. Then,

$$
M_{r}(z, m) \leq K_{d}(\mathbf{m}, \mathbf{l}) \leq M_{s}(z, m),
$$

where $z=\left(z_{1}, z_{2}, \cdots, z_{n}\right)$ with $z_{i}=-\log \left(l_{i} / m_{i}\right)+\left(\left(l_{i} / m_{i}\right)-1\right)$ for $i=1,2, \cdots, n$.

Proof. Taking $f(x)=-\log x, x \in(0, \infty)$, in (53), we obtain (60).

Definition 29. If $\mathbf{1}=\left(l_{1}, l_{2}, \cdots, l_{n}\right)$ and $\mathbf{m}=\left(m_{1}, m_{2}, \cdots, m_{n}\right)$ are two positive probability distributions, then the 
variational distance is defined by

$$
V_{d}(\mathbf{l}, \mathbf{m})=\sum_{i=1}^{n}\left|l_{i}-m_{i}\right| .
$$

Corollary 30. Assume that all the assumptions of Corollary 27 hold. Then,

$$
M_{r}(z, m) \leq V_{d}(\mathbf{l}, \mathbf{m}) \leq M_{s}(z, m)
$$

where $z=\left(z_{1}, z_{2}, \cdots, z_{n}\right)$ with $z_{i}=\left|\left(l_{i} / m_{i}\right)-1\right|-\left(l_{i} / m_{i}\right)+1$ for $i=1,2, \cdots, n$.

Proof. Using the function $f(x)=|x-1|, x \in(0, \infty)$, in (53), we obtain (62).

Definition 31. If $\mathbf{1}=\left(l_{1}, l_{2}, \cdots, l_{n}\right)$ and $\mathbf{m}=\left(m_{1}, m_{2}, \cdots, m_{n}\right)$ are two positive probability distributions, then the Jeffrey distance is defined by

$$
J_{d}(\mathbf{l}, \mathbf{m})=\sum_{i=1}^{n}\left(l_{i}-m_{i}\right) \log \left(\frac{l_{i}}{m_{i z}}\right)
$$

Corollary 32. Assume that all the assumptions of Corollary 27 hold. Then,

$$
M_{r}(z, m) \leq J_{d}(\mathbf{l}, \mathbf{m}) \leq M_{s}(z, m)
$$

where $z=\left(z_{1}, z_{2}, \cdots, z_{n}\right)$ with $z_{i}=\left(\left(l_{i} / m_{i}\right)-1\right) \log \left(l_{i} / m_{i}\right)$ for $i=1,2, \cdots, n$.

Proof. Applying the function $f(x)=(x-1) \log x, x \in(0, \infty)$, in (53), we obtain (64).

Definition 33. If $\mathbf{1}=\left(l_{1}, l_{2}, \cdots, l_{n}\right)$ and $\mathbf{m}=\left(m_{1}, m_{2}, \cdots, m_{n}\right)$ are positive probability distributions, then the Bhattacharyya coefficient is defined by

$$
B_{d}(\mathbf{l}, \mathbf{m})=\sum_{i=1}^{n} \sqrt{l_{i} m_{i}}
$$

Corollary 34. Assume that all the assumptions of Corollary 27 hold, then

$$
M_{r}(z, m) \leq 1-B_{d}(\mathbf{l}, \mathbf{m}) \leq M_{s}(z, m)
$$

where $z=\left(z_{1}, z_{2}, \cdots, z_{n}\right)$ with $z_{i}=-\sqrt{l_{i} / m_{i}}+\left(l_{i} / 2 m_{i}\right)+(1 / 2)$ for $i=1,2, \cdots, n$.

Proof. Using the function $f(x)=-\sqrt{x}, x \in(0, \infty)$, in (53), we obtain (66).

Definition 35. If $\mathbf{l}=\left(l_{1}, l_{2}, \cdots, l_{n}\right)$ and $\mathbf{m}=\left(m_{1}, m_{2}, \cdots, m_{n}\right)$ are positive probability distributions, then the Hellinger distance is defined by

$$
H_{d}(\mathbf{l}, \mathbf{m})=\sum_{i=1}^{n}\left(\sqrt{l_{i}}-\sqrt{m_{i}}\right)^{2}
$$

Corollary 36. Under the assumptions of Corollary 27, the following inequality holds:

$$
M_{r}(z, m) \leq H_{d}(\mathbf{l}, \mathbf{m}) \leq M_{s}(z, m),
$$

where $z=\left(z_{1}, z_{2}, \cdots, z_{n}\right)$ with $z_{i}=\left(\sqrt{l_{i} / m_{i}}-1\right)^{2}$ for $i=1,2$, $\cdots, n$.

Proof. Using the function $f(x)=(\sqrt{x}-1)^{2}, x \in(0, \infty)$, in (53), we obtain (68).

Definition 37. For two positive probability distributions $\mathbf{m}$ $=\left(m_{1}, m_{2}, \cdots, m_{n}\right), \mathbf{l}=\left(l_{1}, l_{2}, \cdots, l_{n}\right)$, the triangular discrimination is defined by

$$
T_{d}(\mathbf{l}, \mathbf{m})=\sum_{i=1}^{n} \frac{\left(l_{i}-m_{i}\right)^{2}}{l_{i}+m_{i}}
$$

Corollary 38. Assume that all the assumptions of Corollary 27 hold, then

$$
M_{r}(z, m) \leq T_{d}(\mathbf{l}, \mathbf{m}) \leq M_{s}(z, m),
$$

where $z=\left(z_{1}, z_{2}, \cdots, z_{n}\right)$ with $z_{i}=\left(l_{i}-m_{i}\right)^{2} /\left(m_{i}\left(l_{i}+m_{i}\right)\right)$ for $i=1,2, \cdots, n$.

Proof. As the function $\phi(x)=(x-1)^{2} /(x+1), x \in(0, \infty)$ is convex. Therefore applying the function $f(x)=\phi(x)$, in (53), we obtain (70).

Now, we begin to give inequalities for Zipf-Mandelbrot entropy. Before presenting the results, we include some introductory part about Zipf-Mandelbrot entropy.

Zipf s law is a fundamental and helpful law in the field of information science. In the field of social studies, this law was regarded as a valuable statistical distribution procedure. The law demonstrate the relation between the size and the rank of discrete phenomena. Furthermore, this law is also applied to the intensity of solar flares, earth quack magnitude, geology, city populations, the size of moon craters, and website traffic etc. It has had limited success in the realm of geology when it comes to assessing petroleum and other mining.

Mandelbrot invented the Zipf-Mandelbrot law in 1966, which is a further enhancement of Zipf's law [28]. The low-rank words in the corpus are taken into account by this law, where $i<100$ [29]: $l(i)=c /(i+\varepsilon)^{s}$, if one put $\varepsilon=0$; the law of Zipf will then be deduced. There are numerous applications of Zipf-Mandelbrot law, which are available in linguistics [29, 30] and information sciences [31] and is also generally useful in ecological field studies [32]. The Zipf- 
Mandelbrot entropy $Z_{e}(\mathfrak{T}, \varepsilon, s)$ is given by

$$
Z_{e}(\mathfrak{T}, \mathcal{\varepsilon}, s)=\frac{s}{\mathfrak{I}_{n, \mathcal{\varepsilon}, s}} \sum_{i=1}^{n} \frac{\log (i+\varepsilon)}{(i+\varepsilon)^{s}}+\log \mathfrak{T}_{n, \varepsilon, s},
$$

where $\varepsilon \geq 0, n \in \mathbb{N}, s>0, \mathfrak{T}_{n, \varepsilon, s}=\sum_{i=1}^{n} 1 /(i+\varepsilon)^{s}$ and the ZipfMandelbrot law is given by: $Z_{M L}(i, n, \varepsilon, s)=\left(1 /(i+\varepsilon)^{s}\right) /$ $\mathfrak{T}_{n, \varepsilon, s}$.

Now, we are in position to give inequalities for ZipfMandelbrot entropy.

Corollary 39. Let $\varepsilon \geq 0, s, m_{i}>0, i=1,2, . ., n$ with $\sum_{i=1}^{n} m_{i}=1$ . If $s \geq 1$ and $r \leq 1$, then

$$
M_{r}(z, m) \leq-Z_{e}(\mathfrak{T}, \varepsilon, s)-\sum_{i=1}^{n} \frac{\log m_{i}}{(i+\varepsilon)^{s} \mathfrak{T}_{n, \varepsilon, s}} \leq M_{s}(z, m)
$$

where $z=\left(z_{1}, z_{2}, \cdots, z_{n}\right)$ with $z_{i}=\left(1 /\left(m_{i}(i+\varepsilon)^{s} \mathfrak{T}_{n, \varepsilon, s}\right)\right) \log$ $\left(1 /\left(m_{i}(i+\varepsilon)^{s} \mathfrak{T}_{n, \mathcal{\varepsilon}, s}\right)\right)-2\left(\left(1 /\left(m_{i}(i+\varepsilon)^{s} \mathfrak{T}_{n, \mathcal{\varepsilon}, s}\right)\right)-1\right)$ for $i=1$, $2, \cdots, n$.

Proof. Let $l_{i}=1 /\left((i+\varepsilon)^{s} \mathfrak{T}_{n, \varepsilon, s}\right), i \in\{1,2, \cdots, n\}$, then

$$
\begin{aligned}
\sum_{i=1}^{n} l_{i} \log l_{i} & =\sum_{i=1}^{n} \frac{1}{(i+\varepsilon)^{s} \mathfrak{T}_{n, \varepsilon, s}} \log \frac{1}{(i+\varepsilon)^{s} \mathfrak{T}_{n, \varepsilon, s}} \\
& =-\sum_{i=1}^{n} \frac{1}{(i+\varepsilon)^{s} \mathfrak{T}_{n, \varepsilon, s}} \log \left((i+\varepsilon)^{s} \mathfrak{T}_{n, \varepsilon, s}\right) \\
& =-\sum_{i=1}^{n} \frac{s}{(i+\varepsilon)^{s} \mathfrak{T}_{n, \varepsilon, s}} \log (i+\varepsilon)-\sum_{i=1}^{n} \frac{\log \mathfrak{T}_{n, \varepsilon, s}}{(i+\varepsilon)^{s} \mathfrak{T}_{n, \varepsilon, s}} \\
& =-\frac{s}{\mathfrak{T}_{n, \varepsilon, s}} \sum_{i=1}^{n} \frac{\log (i+\varepsilon)}{(i+\varepsilon)^{s}}-\frac{\log \mathfrak{T}_{n, \varepsilon, s}}{\mathfrak{T}_{n, \varepsilon, s}} \sum_{i=1}^{n} \frac{1}{(i+\varepsilon)^{s}} \\
& =-Z_{e}(\mathfrak{T}, \varepsilon, s) .
\end{aligned}
$$

Since $\mathfrak{T}_{n, \varepsilon, s}=\sum_{i=1}^{n} 1 /(\varepsilon+i)^{s}, \quad$ therefore, $\sum_{i=1}^{n} 1 /\left((i+\varepsilon)^{s}\right.$ $\left.\mathfrak{\mathfrak { I }}_{n, \varepsilon, s}\right)=1$. Hence, using (59) for $l_{i}=1 /(i+\varepsilon)^{s} \mathfrak{T}_{n, \varepsilon, s}, i=1,2$, .., $n$, we obtain (72).

Utilizing two Zipf s law associated to distinct parameters, we derive estimation for Zipf-Mandelbrot entropy.

Corollary 40. Let $\xi_{1}, \xi_{2} \geq 0, s_{1}, s_{2}>0$. If $s \geq 1$ and $r \leq 1$, then

$$
\begin{aligned}
M_{r}(z, m) & \leq-Z_{e}\left(\mathfrak{T}, \xi_{1}, s_{1}\right)+\sum_{i=1}^{n} \frac{\log \left(\left(i+\xi_{2}\right)^{s_{2}} \mathfrak{T}_{n, \xi_{2}, s_{2}}\right)}{\left(i+\xi_{1}\right)^{s_{1}} \mathfrak{\mathfrak { T }}_{n, \xi_{1}, s_{1}}} \\
& \leq M_{s}(z, m),
\end{aligned}
$$

where $z=\left(z_{1}, z_{2}, \cdots, z_{n}\right)$ with $z_{i}=\left(\left(i+\xi_{2}\right)^{s_{2}} \mathfrak{T}_{n, \xi_{2}, s_{2}}\right) /\left(\left(i+\xi_{1}\right)^{s_{1}}\right.$

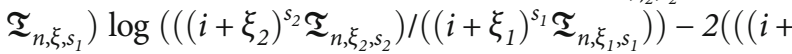
$\left.\left.\left.\xi_{2}\right)^{s_{2}} \mathfrak{T}_{n, \xi_{2}, s_{2}} /(i+\xi)^{s_{1}} \mathfrak{T}_{n, \xi_{1}, s_{1}}\right)-1\right), i=1,2, \cdots, n$.
Proof. Let $l_{i}=1 /\left(i+\xi_{1}\right)^{s_{1}} \mathfrak{T}_{n, \xi_{1}, s_{1}}$ and $m_{i}=1 /\left(i+\xi_{2}\right)^{s_{2}} \mathfrak{T}_{n, \xi_{2}, s_{2}}$, $i=1,2, \cdots, n$, then as in the proof of Corollary 39, we have

$$
\begin{aligned}
\sum_{i=1}^{n} l_{i} \log l_{i} & =\sum_{i=1}^{n} \frac{1}{\left(i+\xi_{1}\right)^{s_{1}} \mathfrak{T}_{n, \xi_{1}, s_{1}}} \log \frac{1}{\left(i+\xi_{1}\right)^{s_{1}} \mathfrak{T}_{n, \xi_{1}, s_{1}}} \\
& =-Z_{e}\left(\mathfrak{T}, \xi_{1}, s_{1}\right), \\
\sum_{i=1}^{n} l_{i} \log m_{i} & =\sum_{i=1}^{n} \frac{1}{\left(i+\xi_{1}\right)^{s_{1}} \mathfrak{T}_{n, \xi_{1}, s_{1}}} \log \frac{1}{\left(i+\xi_{2}\right)^{s_{2} \mathfrak{T}_{n, \xi_{2}, s_{2}}}} \\
& =-\sum_{i=1}^{n} \frac{\log \left(\left(i+\xi_{2}\right)^{s_{2}} \mathfrak{\mathfrak { T }}_{n, \xi_{2}, s_{2}}\right)}{\left(i+\xi_{1}\right)^{s_{1} \mathfrak{T}_{n, \xi_{1}, s_{1}}}} .
\end{aligned}
$$

Also, $\quad \sum_{i=1}^{n} l_{i}=\sum_{i=1}^{n} 1 /\left(i+\xi_{1}\right)^{s_{1}} \mathfrak{T}_{n, \xi_{1}, s_{1}}=1$ and $\sum_{i=1}^{n} m_{i}=$ $\sum_{i=1}^{n} 1 /\left(i+\xi_{2}\right)^{s_{2}} \mathfrak{T}_{n, \xi_{2}, s_{2}}=1$.

Therefore using (59) for $l_{i}=1 /\left(i+\xi_{1}\right)^{s_{1}} \mathfrak{T}_{n, \xi_{1}, s_{1}}$ and $m_{i}=$ $1 /\left(i+\xi_{2}\right)^{s_{2}} \mathfrak{\mathfrak { I }}_{n, \xi_{2}, s_{2}}, i=1,2, \cdots, n$, we obtain (74).

Remark 41. Similarly, we can use (60) and derive inequalities for Zipf-Mandelbrot entropy.

\section{Conclusion}

In the literature, there are several results which are devoted to Jensen's and its related inequalities. In this manuscript, we have used a new approach for the derivation of improvements of Jensen's inequality. We have given Jensen's inequality and its improvements in quantum calculus. In particular, we have deduced Hermite-Hadamard type inequalities for $q$-integrals. We have also given applications of main results for some well-known inequalities and in information theory. The results of this manuscript which are initiated and given in quantum calculus may stimulate further research.

\section{Data Availability}

No data were used to support this study.

\section{Conflicts of Interest}

The authors declare that there are no conflicts of interest regarding the publication of this paper.

\section{Authors' Contributions}

All authors contributed equally and significantly in this paper. All authors read and approved the final manuscript.

\section{References}

[1] P. Cerone and S. S. Dragomir, Mathematical Inequalities, CRC Press, Boca Raton, 2010.

[2] J. E. Pečarić and F. Proschan, Convex Functions, Partial Orderings and Statistical Applications, Academic Press, Inc., 1992. 
[3] M. Adil Khan, S. Khan, and Y.-M. Chu, "A new bound for the Jensen gap with applications in information theory," IEEE Access, vol. 8, pp. 98001-98008, 2020.

[4] Q. Lin, “Jensen inequality for superlinear expectations," Statistics \& Probability Letters, vol. 151, pp. 79-83, 2019.

[5] M. J. Cloud, B. C. Drachman, and L. P. Lebedev, Inequalities with Applications to Engineering, Cham Heidelberg New York Dordrecht London, Springer, 2014.

[6] C. C. White and D. P. Harrington, "Application of Jensen's inequality to adaptive suboptimal design," Journal of Optimization Theory and Applications, vol. 32, no. 1, pp. 89-99, 1980.

[7] S. A. Azar, "Jensen's inequality in finance," International Advances in Economic Research, vol. 14, no. 4, pp. 433-440, 2008.

[8] N. Mukhopadhyay, "On sharp Jensen's inequality and some unusual Applications," Communications in Statistics-Theory and Methods, vol. 40, no. 7, pp. 1283-1297, 2011.

[9] N. Tapus and P. G. Popescu, "A new entropy upper bound," Applied Mathematics Letters, vol. 25, no. 11, pp. 1887-1890, 2012.

[10] J. Pečarić and J. Perić, "Refinements of the integral form of Jensen's and the Lah-Ribarič inequalities and applications for Csiszár divergence," Journal of Inequalities and Applications, vol. 2020, no. 1, Article ID 108, 2020.

[11] D. Costarelli and R. Spigler, "How sharp is the Jensen inequality?," Journal of Inequalities and Applications, vol. 2015, no. 1, Article ID 69, 2015.

[12] Đ. Pečarić, J. Pečarić, and M. Rodić, “About the sharpness of the Jensen inequality," Journal of inequalities and applications, vol. 2018, no. 1, Article ID 337, 2018.

[13] S. Khan, M. Adil Khan, S. I. Butt, and Y.-M. Chu, "A new bound for the Jensen gap pertaining twice differentiable functions with applications," Advances in Difference Equations, vol. 2020, no. 1, Article ID 333, 2020.

[14] M. Adil Khan, S. Khan, Đ. Pečarić, and J. Pečarić, "New improvements of Jensen's type inequalities via 4-convex functions with applications," Revista de la Real Academia de Ciencias Exactas, Físicas y Naturales. Serie A. Matemáticas, vol. 115, no. 2, article 43, pp. 1-21, 2021.

[15] K. A. Khan, T. Niaz, Đ. Pečarić, and J. Pečarić, "Estimation of different entropies via Hermite interpolating polynomial using Jensen type functionals," The Journal of Analysis, vol. 29, no. 1, pp. 15-46, 2021.

[16] T. Niaz, K. A. Khan, Đ. Pečarić, and J. Pečarić, “Estimation of different entropies via Taylor one point and Taylor two points interpolations using Jensen type functionals," International Journal of Analysis and Applications, vol. 17, no. 5, pp. 686710, 2019.

[17] K. A. Khan and J. Pečarić, "mixed symmetric means related to the classical Jensen's inequality," Journal of Mathematical Inequalities, vol. 7, no. 1, pp. 43-62, 2013.

[18] L. Horváth, K. A. Khan, and J. Pečarić, "Combinatorial improvements of Jensen's inequality, Monographs in inequalities," Element, Zagreb, vol. 8, p. 229, 2014.

[19] T. Ernst, The History of q-Calculus and New Method, Department of Mathematics, Uppsala University, Sweden, 2000.

[20] A. Bokulich and G. Jaeger, Philosophy of Quantum Information and Entanglement, Cambridge Uniersity Press, Cambridge, UK, 2010.
[21] H. Kalsoom, J.-D. Wu, S. Hussain, and M. A. Latif, “Simpson's type inequalities for co-ordinated convex functions on quantum calculus," Symmetry, vol. 11, no. 6, article 768, 2019.

[22] M. Arif, H. M. Srivastava, and S. Umar, "Some applications of a q-analogue of the Ruscheweyh type operator for multivalent functions," Revista de la Real Academia de Ciencias Exactas, Físicas y Naturales. Serie A. Matemáticas, vol. 113, no. 2, pp. 1211-1221, 2019.

[23] M. Arif, O. Barkub, H. M. Srivastava, S. Abdullah, and S. A. Khan, "Some Janowski type harmonic q-starlike functions associated with symmetrical Points," Mathematics, vol. 8, no. 4 , article $629,2020$.

[24] H. M. Srivastava, M. Arif, and M. Raza, "Convolution properties of meromorphically harmonic functions defined by a generalized convolution q-derivative operator," Aims Mathematics, vol. 6, no. 6, pp. 5869-5885, 2021.

[25] J. Tariboon and S. K. Ntouyas, "Quantum calculus on finite intervals and applications to impulsive difference equations," Advances in Difference Equations, vol. 2013, no. 1, Article ID 282, 2013.

[26] N. Alp, M. Z. Sarıkaya, M. Kunt, and İ. İşcan, " $q$-Hermite Hadamard inequalities and quantum estimates for midpoint type inequalities via convex and quasi-convex functions," Journal of King Saud University-Science, vol. 30, no. 2, pp. 193-203, 2018.

[27] S. Marinković, P. Rajković, and M. Stanković, "The inequalities for some types of $q$-integrals," Computers \& Mathematics with Applications, vol. 56, no. 10, pp. 2490-2498, 2008.

[28] B. Mandelbrot, "Information theory and psycholinguistics: a theory of words frequencies," in Reading in Mathematical Social Scence, P. Lazafeld and N. Henry, Eds., MIT Press, Cambridge MA, 1966.

[29] M. A. Montemurro, "Beyond the Zipf-Mandelbrot law in quantitative linguistics," Physica A: Statistical Mechanics and its Applications, vol. 300, no. 3-4, pp. 567-578, 2001.

[30] D. Manin, "Mandelbrot's model for Zipf's law: can Mandelbrot's model explain Zipf's law for language," Journal of Quantitative Linguistics, vol. 16, no. 3, pp. 274-285, 2009.

[31] Z. K. Silagadze, "Citations and the Zipf-Mandelbrot law," Complex Systems, vol. 11, pp. 487-499, 1997.

[32] D. Mouillot and A. Lepretre, "Introduction of relative abundance distribution (RAD) indices, estimated from the rank-frequency diagrams (RFD), to assess changes in community diversity," Environmental Monitoring and Assessment, vol. 63, no. 2, pp. 279-295, 2000. 